\title{
Eletrorretinograma multifocal em pacientes tratadas com tamoxifeno em baixa dosagem
}

\author{
Multifocal ERG in patients treated with low-dose tamoxifen
}

\author{
Adriana Berezovsky ${ }^{1}$ \\ Solange Rios Salomão \\ Josenilson Martins Pereira ${ }^{3}$ \\ Paula Yuri Sacai ${ }^{4}$ \\ Márcia Maria Motono Chojniak 5
}

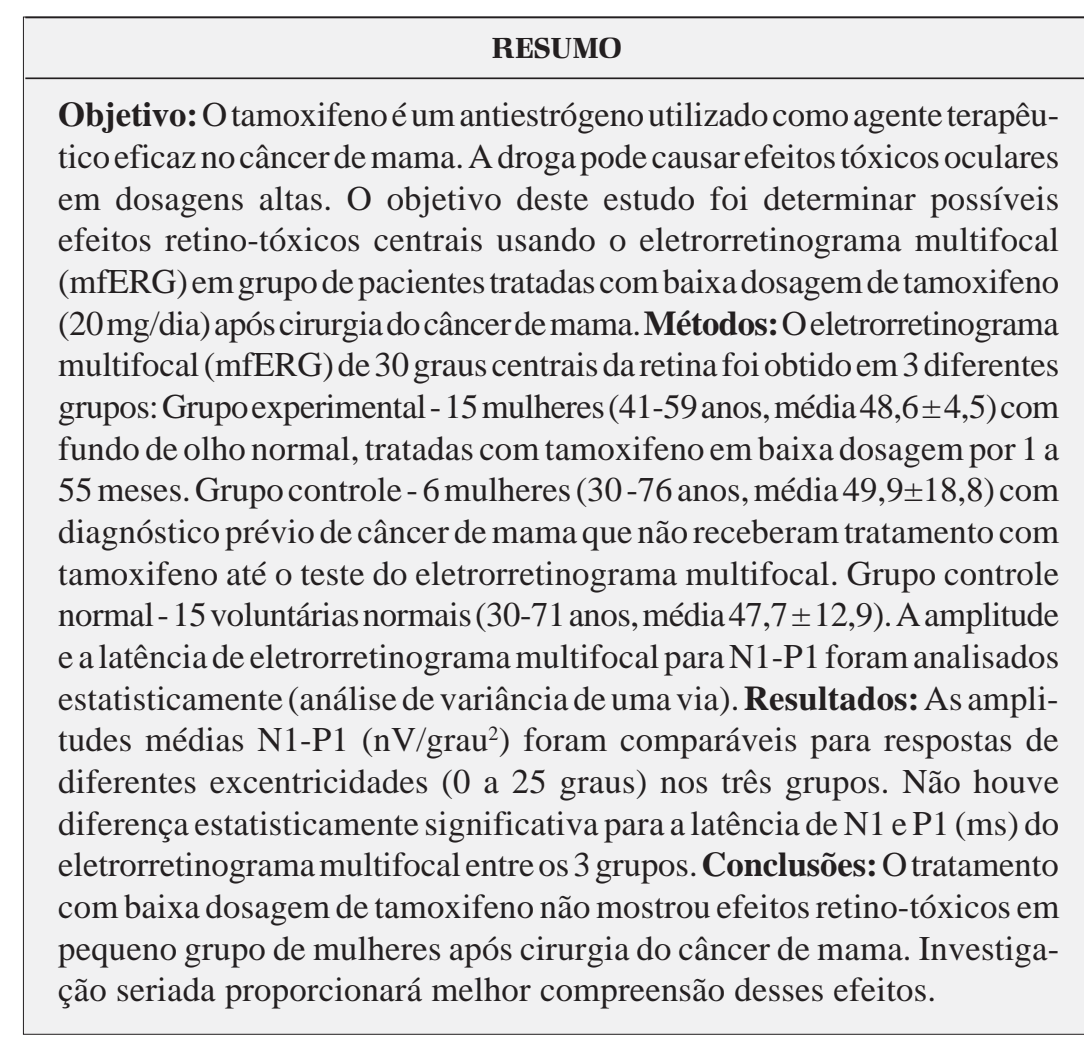

Descritores: Neoplasias mamárias/quimioterapia; Tamoxifeno/efeitos adversos; Tamoxifeno/administração \& dosagem; Tamoxifeno/toxicidade; Retina/efeito de drogas; Eletrorretinografia.
Universidade Federal de São Paulo - Depto de Oftalmologia - Rua Botucatu, 822 - CEP 04023-062 São Paulo (SP)

${ }^{1}$ Professora Adjunta do Departamento de Oftalmologia e Doutora em Ciências pela Universidade Federal de São Paulo (UNIFESP).

2 Professora Adjunta do Departamento de Oftalmologia, Doutora em Ciências e Chefe do Setor de Eletrofisiologia Visual Clínica do Departamento de Oftalmologia da Universidade Federal de São Paulo (UNIFESP).

3 Tecnólogo Oftálmico do Departamento de Oftalmologia da Universidade Federal de São Paulo (UNIFESP). 4 Tecnóloga Oftálmica do Departamento de Oftalmologia da Universidade Federal de São Paulo (UNIFESP).

${ }^{5}$ Médica oftalmologista do Hospital do Câncer - AC. Camargo e Doutora em Medicina pela Universidade Federal de São Paulo (UNIFESP).

Endereço para correspondência: Adriana Berezovsky Depto de Oftalmologia - UNIFESP - R. Botucatu, 822 CEP 04023-062 - São Paulo (SP)

E-mail: aberezovsky@oftalmo.epm.br

Recebido para publicação em 04.07.2003

Versão revisada recebida em 11.12.2003

Aprovação em 20.01.2004

Nota Editorial: Pela análise deste trabalho e por sua anuência na divulgação desta nota, agradecemos ao Dr. Walter Yukihiko Takahashi.

\section{INTRODUÇÃO}

O tamoxifeno é uma droga antiestrógena amplamente utilizada como agente terapêutico eficaz em pacientes submetidos à cirurgia do câncer de mama, com o propósito de reduzir a recidiva e a ocorrência de tumor na mama contralateral ${ }^{(1-2)}$. A estrutura química do tamoxifeno é similar à de outras drogas bem conhecidas como causadoras de toxicidade ocular, como a cloroquina e a imipramina. O primeiro estudo sobre toxicidade retiniana provocada pelo uso de tamoxifeno mostrou redução significante da acuidade visual em 3 de 4 pacientes tratados com essa $\operatorname{droga}^{(3)}$. A diminuição da acuidade visual foi relacionada à retinopatia, primariamente afetando a mácula e acompanhada por edema macular.

Manifestações oculares tais como opacidades corneanas superficiais, diminuição da acuidade visual e evidência de uma retinopatia com pequenos pontos brancos, lesões no epitélio pigmentado da retina e disfunção foveal, 
foram descritas em estudos com altas dosagens da droga (60$100 \mathrm{mg} / \mathrm{m}^{2}$ ) após uso por mais de um ano ${ }^{(3-6)}$. Defeitos de campo visual, como constrição periférica e escotoma central, têm sido relatados $^{(5-6)}$. No entanto, existe controvérsia sobre os efeitos do fármaco na retina quando administrado em baixas doses (20 mg/dia). Não há relatos de anormalidades oculares após 3 a 4 meses do uso de tamoxifeno em baixas $\operatorname{doses}^{(5)}$ e a retinopatia foi diagnosticada somente após 9 a 14 meses de uso ${ }^{(7)}$.

Há relato isolado de um caso de neurite óptica bilateral após tratamento com tamoxifeno ${ }^{(8)}$.

A incidência de 6,3\% de toxicidade ocular foi demonstrada em um estudo prospectivo de 63 pacientes que receberam tamoxifeno em baixa dosagem após tratamento com duração média de 25 meses $^{(9)}$. A prevalência de toxicidade ocular em pacientes visualmente assintomáticos tratados com tamoxifeno é de aproximadamente $1,5 \%{ }^{(10)}$. Enquanto a incidência de retinopatia parece baixa em pacientes que receberam tamoxifeno em baixas doses, ainda não está esclarecido se mudanças no ERG podem preceder alterações fundoscópicas.

A eletrorretinografia multifocal (mfERG) é uma técnica de registro complexo dos potenciais elétricos locais originários de múltiplas áreas da retina em resposta à estimulação luminosa. O mfERG tem sido amplamente utilizado como método diagnóstico de doenças como maculopatias, toxicidade retiniana, diabetes entre outras ${ }^{(11)}$. Anormalidades no mfERG podem preceder alterações de fundo de olho ${ }^{(12)}$.

O objetivo deste estudo foi avaliar a função retiniana central pelo mfERG, determinando possíveis efeitos tóxicos na retina causados pelo tratamento com baixa dosagem de tamoxifeno, em pacientes tratados após cirurgia do câncer de mama.

\section{MÉTODOS}

\section{Participantes}

O mfERG foi registrado em 36 mulheres, após obtenção do consentimento livre e esclarecido. Este protocolo de pesquisa foi aprovado pelo Comitê de Ética em Pesquisa da UNIFESP. Os critérios de inclusão foram: acuidade visual com a melhor correção óptica $=20 / 25$ medida para longe $(4 \mathrm{~m})$ pela tabela ETDRS, fundo de olho normal, ausência de queixas visuais e de história familiar de doenças oculares hereditárias e bom estado geral de saúde. Os participantes foram divididos em 3 diferentes grupos. Grupo Experimental - 15 mulheres (idade de

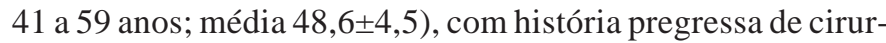
gia do câncer de mama, tratadas com tamoxifeno (20mg/dia) por 1 a 55 meses (média 23,6 14,4 meses; mediana 26 meses). Somente mulheres receberam tratamento com duração inferior a 12 meses ( 1 , 3 e 5 meses). Grupo Controle - 6 mulheres (idade de 30 a 76 anos, média 49,9₫18,8) com história pregressa de cirurgia do câncer de mama, que ainda não receberam tratamento com tamoxifeno até o teste do mfERG. Grupo Controle Normal - 15 voluntárias saudáveis normais (idade de 30 a 71 anos, média $47,7 \pm 12,9$ ).

\section{Procedimento}

Após a medida monocular da acuidade visual com a melhor correção óptica, o mfERG foi registrado num olho escolhido ao acaso, captado por eletrodo corneano bipolar em forma de lente de contato (GoldLens, Doran Instruments, MD, USA). Após a dilatação mínima de $6 \mathrm{~mm}$ da pupila com uma gota de tropicamida a $1 \%$ e uma gota de fenilefrina a $10 \%$, foi instilada uma gota de colírio de metilcelulose a $2 \%$ na cúpula da lente para proteção da córnea. Um eletrodo terra foi colocado no lobo da orelha ipsilateral, preenchido com gel eletrolítico.

Áreas retinianas múltiplas mediadas pelo mfERG foram obtidas com o sistema eletrodiagnóstico multifocal (VERIS 4.9; Electro-Diagnostic Imaging, San Mateo, CA, EUA). O estímulo utilizado foi um conjunto de 103 hexágonos subtendendo uma área retiniana de $46^{\circ}$ de diâmetro horizontal e $39^{\circ}$ vertical. A área dos hexágonos foi escalada com excentricidade para obter respostas de mfERGs de amplitudes similares em 5 anéis concêntricos, com intervalos de cinco graus a partir de um único hexágono central. Uma mira em forma de " $X$ “" central foi usada para fixação. A luminância dos estímulos hexagonais pretos $\left(0,45 \mathrm{~cd} / \mathrm{m}^{2}\right)$ e brancos $\left(280 \mathrm{~cd} / \mathrm{m}^{2}\right)$ foi revertida de acordo com uma seqüência-m binária ${ }^{(13)}$. Os estímulos foram dispostos em uma câmara/refrator CRT de alta luminância monocromática com uma taxa de reversão de $75 \mathrm{~Hz}$, totalizando 16 segmentos de 27 segundos de duração (aproximadamente 8 minutos de exame). A câmara/refrator foi usada para compensar o erro refrativo do paciente e monitorar a posição do olho, o alinhamento do eletrodo de lente de contato com a pupila e a estabilidade da fixação durante o registro. Diante de qualquer perda de fixação, o segmento foi interrompido e descartado. As respostas retinianas foram amplificadas com ganho de 50.000 e filtros eletrônicos de 10 e $300 \mathrm{~Hz}$.

Os parâmetros de amplitude pico-a-pico (nV/grau²) das ondas N1-P1 e de latência (ms) de N1 (primeira deflexão negativa) e de P1 (primeira deflexão positiva) foram calculados para anéis de excentricidade compreendendo os $30^{\circ}$ centrais do campo visual.

\section{MÉTODO ESTATÍSTICO}

A análise de variância de uma via (one-way ANOVA) foi usada para comparação estatística dos parâmetros de amplitude e a latência do mfERG.

\section{RESULTADOS}

As amplitudes médias N1-P1 foram comparáveis para respostas de diferentes excentricidades ( 0 a 25 graus) no grupo de pacientes que receberam o tamoxifeno, o grupo de pacientes submetidos à cirurgia de câncer de mama e não iniciaram o uso de tamoxifeno e o grupo de voluntárias normais, como mostra a figura 1 . A tabela 1 mostra a média dos valores das amplitudes de N1-P1 nas áreas de excentricidades de $0^{\circ}$ a $25^{\circ}$ nos três grupos estudados. 
Não houve diferença estatisticamente significativa para o tempo de culminação de N1 e P1 (ms) dos mfERGs nos 3 grupos como mostram as figuras 2 e 3 .

A tabela 2 mostra a média dos valores do tempo de culminação de N1 e P1 (ms) dos mfERGs nas áreas de excentricidades de $0^{\circ}$ a $25^{\circ}$ para os grupos de pacientes que receberam o tamoxifeno, o grupo de pacientes que foram submetidos à cirurgia de câncer de mama e não iniciaram o uso de tamoxifeno e o grupo de voluntárias normais.

A figura 4 mostra um exemplo representativo de mfERG de OD de paciente do sexo feminino, com idade de 41 anos tratada com baixa dosagem de tamoxifeno por 30 meses.

\section{DISCUSSÃO}

O presente estudo mostrou que não houve efeitos retinotóxicos centrais causados pelo tamoxifeno usado em baixa dosagem em um pequeno grupo de pacientes tratados após cirurgia do câncer de mama.

Na literatura não está claro se anormalidades nos achados eletrorretinográficos podem preceder a retinopatia observável

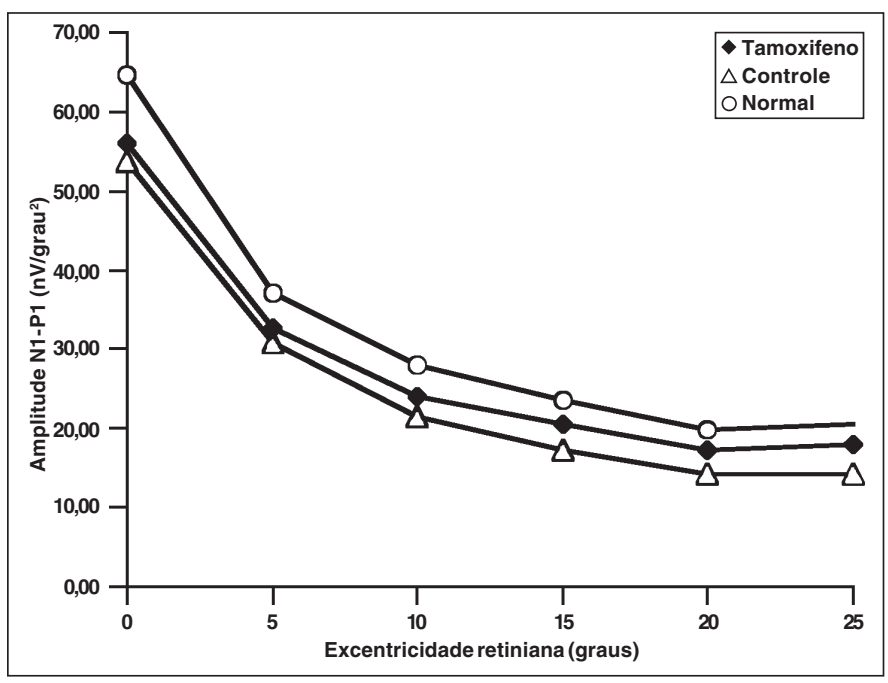

Figura 1 - Amplitude (nV/grau ${ }^{2}$ nas 5 diferentes áreas de excentricidade de 0 a 25 graus em intervalos de 5 graus. (anéis 1, 2, 3, 4 e 5 correspondendo $0,5,10,15,20,25$ graus)

\begin{tabular}{|c|c|c|c|c|c|c|c|}
\hline & $\begin{array}{l}\text { Amp N1-P1 } \\
\left(\mathrm{nV} / \mathrm{grau}^{2}\right)\end{array}$ & $0^{\circ}$ & $5^{\circ}$ & $10^{\circ}$ & $15^{\circ}$ & $20^{\circ}$ & $25^{\circ}$ \\
\hline \multirow[t]{2}{*}{ Tamoxifeno } & Média & 53,79 & 31,29 & 22,84 & 19,66 & 16,57 & 17,35 \\
\hline & DP & 16,07 & 10,65 & 8,12 & 7,47 & 6,29 & 6,88 \\
\hline \multirow[t]{2}{*}{ Controle } & Média & 49,07 & 28,82 & 19,65 & 16,20 & 13,32 & 13,35 \\
\hline & DP & 21,16 & 11,29 & 9,73 & 7,73 & 5,63 & 5,45 \\
\hline \multirow[t]{2}{*}{ Normal } & Média & 64,56 & 37,06 & 28,04 & 23,59 & 19,89 & 20,42 \\
\hline & DP & 15,95 & 9,10 & 7,86 & 6,52 & 5,21 & 5,26 \\
\hline
\end{tabular}

em pacientes com diagnóstico prévio de câncer de mama e que receberam baixa dosagem de tamoxifeno (20 mg/dia). Uma pequena redução nas amplitudes fotópicas e escotópicas do ERG de campo total foi descrita em uma paciente que recebeu alta dosagem de tamoxifeno para tratamento de câncer de mama metastático ${ }^{(5)}$. Dois outros casos relatados mostraram ERGs normais em pacientes com alta dosagem de tamoxifeno ${ }^{(14-15)}$. Estudos prévios mostraram que anormalidades retinianas podem ocorrer após no mínimo um ano de tratamento com tamoxifeno. Em nosso estudo nenhuma paciente que recebeu baixa dosagem de tamoxifeno de 1 a 55 meses demonstrou alteração na eletrorretinografia multifocal.

Até o momento, não existem relatos de trabalhos na literatura utilizando a técnica da eletrorretinografia multifocal em pacientes tratados com tamoxifeno após cirurgia de câncer de mama. Um

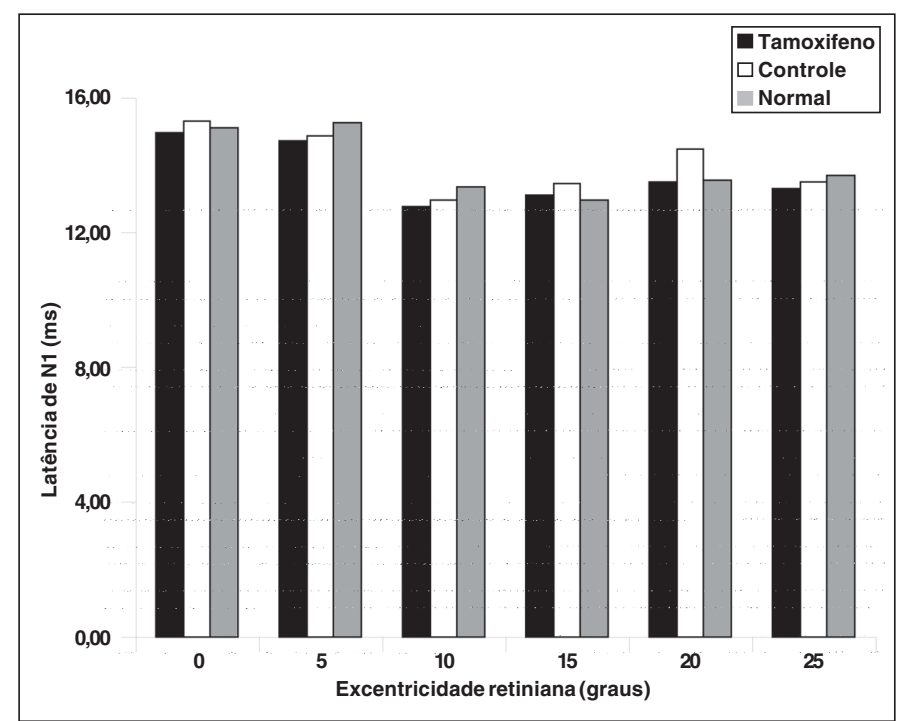

Figura 2 - Latência de N1 (ms) dos três grupos estudados

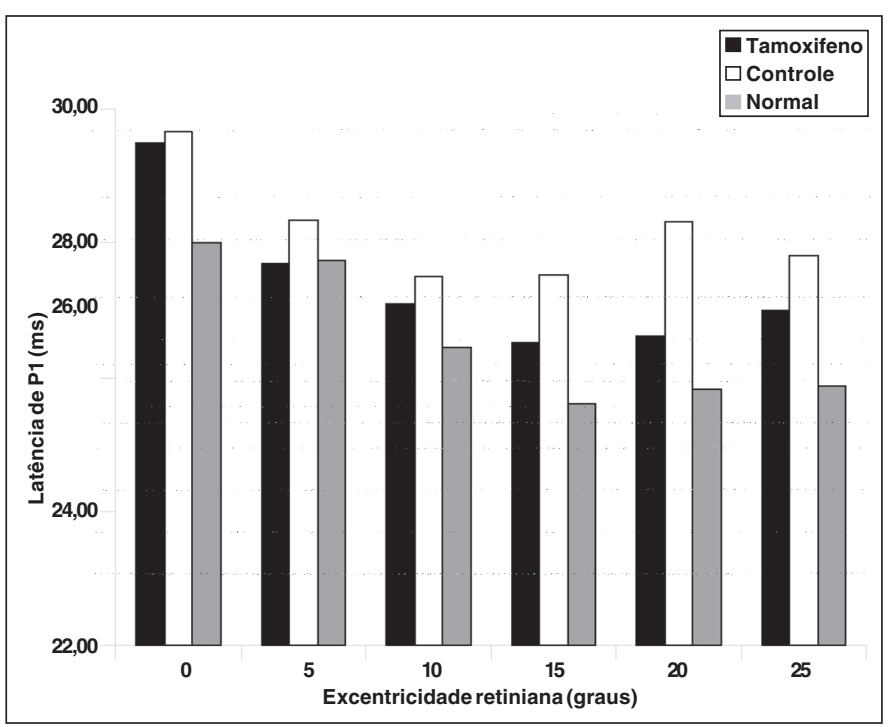

Figura 3- Latência de P1 (ms) dos três grupos estudados 


\begin{tabular}{|c|c|c|c|c|c|c|c|c|c|c|c|c|}
\hline \multirow[b]{2}{*}{ Latência } & \multicolumn{2}{|c|}{$0^{\circ}$} & \multicolumn{2}{|c|}{$5^{\circ}$} & \multicolumn{2}{|c|}{$10^{\circ}$} & \multicolumn{2}{|c|}{$15^{\circ}$} & \multicolumn{2}{|c|}{$20^{\circ}$} & \multicolumn{2}{|c|}{$25^{\circ}$} \\
\hline & N1 & P1 & N1 & P1 & N1 & P1 & N1 & P1 & N1 & P1 & N1 & P1 \\
\hline \multicolumn{13}{|c|}{ Tamoxifeno } \\
\hline Média & 15,13 & 29,92 & 13,01 & 27,64 & 12,89 & 27,06 & 13,28 & 27,06 & 13,28 & 27,06 & 13,42 & 27,38 \\
\hline DP & 1,94 & 2,42 & 1,07 & 3,05 & 1,96 & 3,44 & 1,74 & 3,37 & 1,74 & 3,37 & 0,74 & 3,16 \\
\hline \multicolumn{13}{|l|}{ Controle } \\
\hline Média & 15,83 & 30,13 & 14,88 & 28,75 & 13,18 & 28,20 & 13,32 & 28,35 & 14,17 & 28,88 & 13,62 & 28,47 \\
\hline DP & 1,48 & 1,94 & 0,97 & 1,54 & 0,63 & 3,80 & 2,58 & 4,27 & 2,68 & 3,73 & 1,45 & 3,56 \\
\hline \multicolumn{13}{|l|}{ Normal } \\
\hline Média & 15,11 & 28,01 & 15,27 & 27,73 & 13,37 & 26,45 & 12,99 & 25,61 & 13,55 & 25,83 & 20,42 & 13,72 \\
\hline DP & 1,63 & 1,54 & 1,12 & 0,96 & 0,67 & 0,97 & 0,52 & 0,96 & 0,68 & 0,99 & 5,26 & 0,78 \\
\hline
\end{tabular}

a

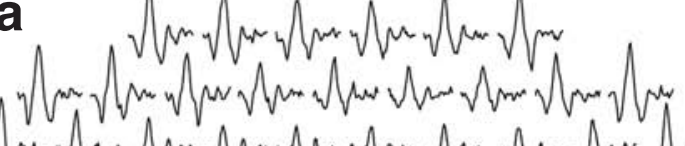

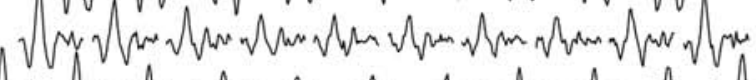

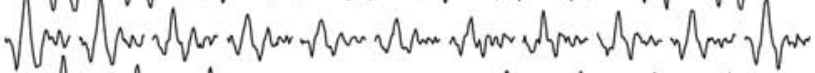

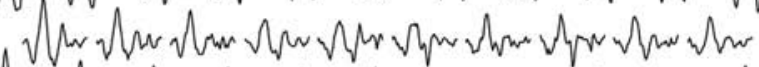

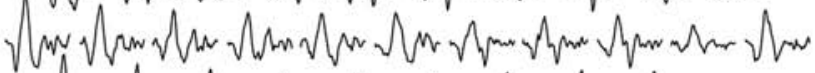

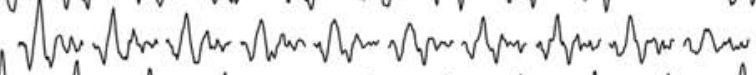

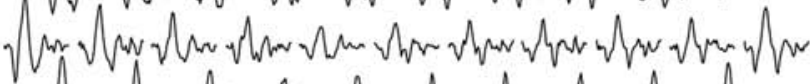

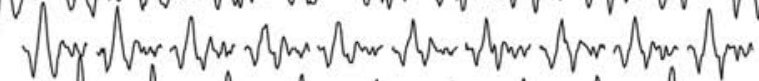

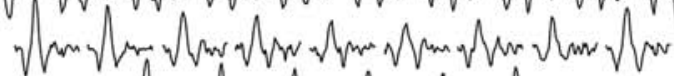

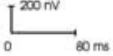

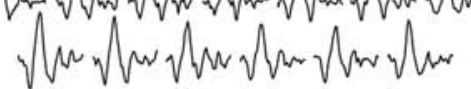

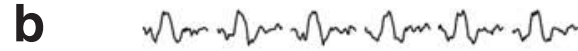

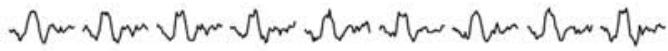

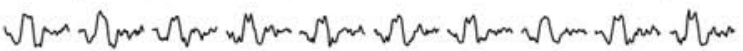

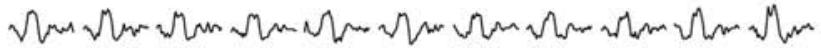

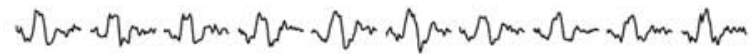

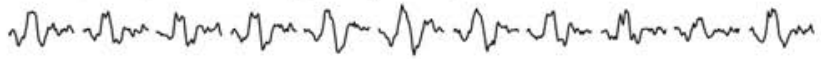

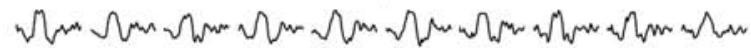

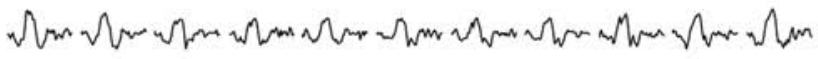

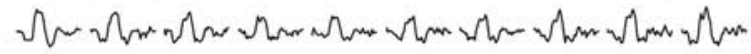
$\mathrm{L}_{\mathrm{L}}^{200 \mathrm{miv}}$

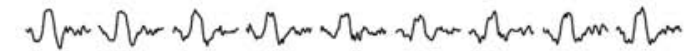

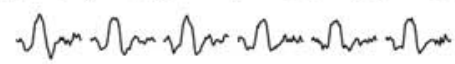

Total Reiponese
$7.90 \mathrm{nV} / \mathrm{deg} / 2$
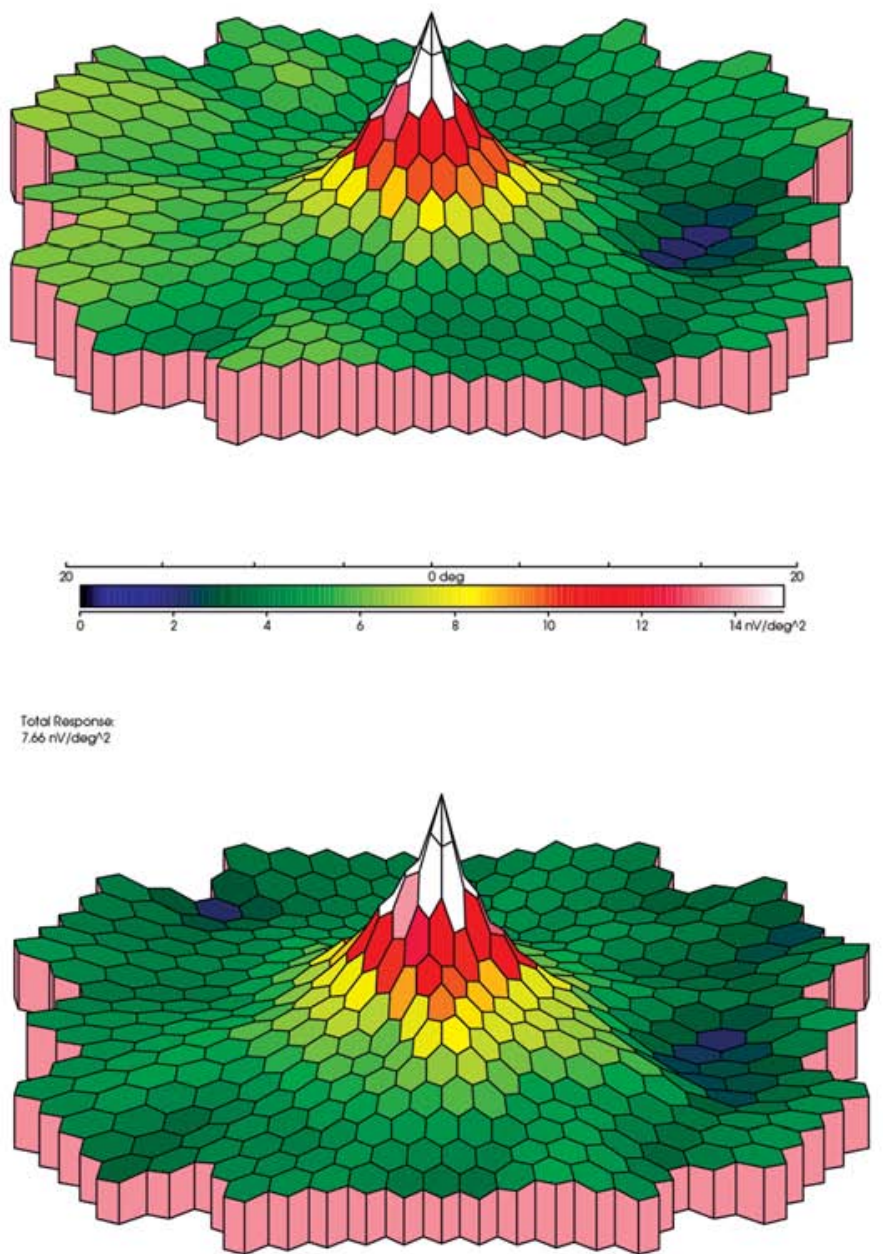

Figura 4 - a) Conjunto de traçados e representação topográfica funcional do mfERG do olho direito de uma paciente de 49 anos tratada com baixa dosagem de tamoxifeno tratada por 30 meses. b) Conjunto de traçados e representação topográfica funcional do mfERG do olho direito de uma voluntária normal de 45 anos de idade 
relato de caso mostrou redução das respostas do mfERG em mulher de 84 anos de idade com diagnóstico prévio de câncer de mama e com queixa de redução da acuidade visual ${ }^{(16)}$.

Nossos resultados sugerem que não há evidência de toxicidade causada pelo uso do tamoxifeno devido à disfunção dos cones centrais. O aumento do número de casos, principalmente no grupo controle que ainda não tomou a medicação é importante para ampliar e estender os presentes achados.

\section{CONCLUSÕES}

Os achados eletrorretinográficos multifocais mostraram que baixa dosagem de tamoxifeno (20 mg) não mostrou efeitos retino-tóxicos em um grupo de 15 mulheres após cirurgia do câncer de mama. Investigação seriada proporcionará uma melhor compreensão destes efeitos.

\section{ABSTRACT}

Purpose: Tamoxifen, an antiestrogen, has been used as an effective therapeutic agent in the treatment of breast cancer. The drug has been shown to cause ocular toxic effects. The purpose of this study was to determine possible central retinal toxicity by multifocal electroretinograms (mfERGs) in a cohort of patients treated with low-dose tamoxifen (20 mg/day) for breast cancer. Methods: Multifocal electroretinograms of the central 30 degrees of the retina were obtained of 3 different groups: Experimental group - 15 females (41-59 years, mean $48.6 \pm 4.5$ ) with normal fundus, treated with low-dose tamoxifen for 1 to 55 months. Control group - 6 females (30-76 years, mean $49.9 \pm 18.8$ ) with previous breast cancer diagnosis who had not received tamoxifen as treatment until ERG testing. Normal control group - 15 normal female volunteers (30-71 years, mean $47.7 \pm 12.9$ ). The multifocal amplitude and latency for N1-P1 were measured and statistically analyzed (one-way ANOVA). Results: Mean amplitudes N1-P1 (nV/deg ${ }^{2}$ ) were comparable for responses of different eccentricity (0 to 25 degrees). There was no statistically significant difference for implicit time of $\mathrm{N} 1$ and P1 (ms) in multifocal electroretinograms between the 3 groups. Conclusions: Low-dose tamoxifen showed no retinotoxic effect in this small group of women with breast cancer. Follow-up investigation could provide a better understanding of these effects.

Keywords: Breast neoplasms/drug therapy; Tamoxifen/ adverse effects; Tamoxifen/administration \& dosage; Retina/ drug effects; Electroretinography

\section{REFERÊNCIAS}

1. Furr BJA, Jordan VC. The pharmacology and clinical uses of tamoxifen. Pharmacol Ther. 1984;25(2):127-205.

2. Feitosa FEL, Juaçaba SF, Medeiros FC. Alterações endometriais em pacientes com câncer de mama tratados com tamoxifeno. Rev Bras Ginecol Obstet. 2002;24(4):233-9.

3. Kaiser-Kupfer MI, Lippman ME. Tamoxifen retinopathy. Cancer Treat Rep. 1978;62(3):315-20.

4. Kaiser-Kupfer MI, Kupfer C, Rodrigues MM. Tamoxifen retinopathy: a clinicopathologic report. Ophthalmology. 1981;88(1):89-93.

5. Beck M, Mills PV. Ocular assessment of patients treated with tamoxifen. Cancer Treat Rep. 1979;63(11-12):1833-4.

6. McKeown CA, Swartz M, Blom J, Maggiano JM. Tamoxifen retinopathy. Br J Ophthalmol. 1981;65(3):177-9.

7. Pugesgaard T, Von Eyben FE. Bilateral optic neuritis evolved during tamoxifen treatment. Cancer. 1986;58(2):383-6.

8. Vinding T, Nielsen NV. Retinopathy caused by treatment with tamoxifen in low dosage. Acta Ophthalmol. (Copenh) 1983;61(1):45-50.

9. Pavlidis NA, Petris C, Briassoulis E, Klouvas G, Psilas C, Rempapis J, et al. Clear evidence that long-term, low-dose tamoxifen treatment can induce ocular toxicity. A prospective study of 63 patients. Cancer. 1992;69(12):2961-4.

10. Heier JS, Dragoo RA, Enzenauer RW, Waterhouse WJ. Screening for ocular toxicity in asymptomatic patients treated with tamoxifen Am J Ophthalmol. 1994;117(6):772-5. Comment on: Am J Ophthalmol. 1995;119(1):112-3.

11. Marmor MF, Hood DC, Keating D, Kond M, Seeliger MW, Miyake Y. Guidelines for basic Multifocal Electroretinography (mfERG). Doc Ophthalmol. 2003;106(2):105-15. Erratum in: Doc Ophthalmol. 2003;106(3):338.

12. Kretschmann U, Bock M, Gockeln R, Zrenner E. Clinical applications of multifocal electroretinography. Doc Ophthalmol. 2000;100(2-3):99-113.

13. Sutter EE. Imaging visual function with the multifocal $\mathrm{m}$ - sequence technique. Vision Res. 2000;41(10-11):1241-55.

14. Gerner EW. Ocular toxicity of tamoxifen. Ann Ophthalmol 1989;21(11):420-3.

15. Bentley CR, Davies G, Aclimandos WA. Tamoxifen retinopathy: a rare but serious complication. BMJ. 1992;304(6825):495-6.

16. Sadowski B, Kriegbaum C, Apfelstedt-Sylla E. Tamoxifen side effects, agerelated macular degeneration(AMD) or cancer associated retinopathy (CAR)?. Eur J Ophthalmol. 2001;11(3):309-12.

\title{
ABO ELETRÔNICO
}

\author{
Novo site
}

\section{Acesso: http://www.abonet.com.br}

